\title{
Error estimates for elasto-plastic problems
}

RAIRO - Analyse numérique, tome 11, no 2 (1977), p. 135-144.

$<$ http://www.numdam.org/item?id=M2AN_1977_11_2_135_0>

(C) AFCET, 1977, tous droits réservés.

L'accès aux archives de la revue « RAIRO - Analyse numérique » implique l'accord avec les conditions générales d'utilisation (http://www.numdam.org/ legal.php). Toute utilisation commerciale ou impression systématique est constitutive d'une infraction pénale. Toute copie ou impression de ce fichier doit contenir la présente mention de copyright.

\section{Numdam}

Article numérisé dans le cadre du programme

Numérisation de documents anciens mathématiques

http://www.numdam.org/ 


\title{
ERROR ESTIMATES \\ FOR ELASTO-PLASTIC PROBLEMS ( ${ }^{1}$ )
}

\author{
by Richard S. FALK $\left({ }^{*}\right)\left({ }^{2}\right)$ and Bertrand MERCIER $\left({ }^{3}\right)$ \\ Communiqué par P. G. Ciarlet
}

\begin{abstract}
Under some reasonable smoothness assumptions on the displacements, we are able to derive an error estimate of the form $\left\|\sigma-\sigma_{h}\right\|_{L^{2}(\Omega)} \leqslant C h$, for the approximation of the stress field $\sigma$ in some problems in elasto-plasticity.

Using the same ideas, we also find a piecewise linear approximation of Mosolov's problem, for which we still get an $0(h)$ error estimate.
\end{abstract}

\section{INTRODUCTION}

In this paper, we consider the approximation of some stationary elastic-perfectly problems formalized by Duvaut-Lions [7]. Our main purpose is to derive error estimates for the approximation of the stress field $\sigma$ given by a finite element method, appearing in Mercier [16]. The approximate problems we solve, however, will be in terms of the displacements, which are the natural variables for computation.

This work appears to parallel that of Johnson [12], who considered the derivation of error estimates for evolution problems in plasticity. In this stationary case, we are able to obtain improved error estimates over those derived in [12].

Using some ideas from Johnson [11], we are able to establish the existence of a displacement in $L^{\gamma}(\Omega)$ for a class of problems in stationary elasto-plasticity.

Finally, we apply the method to obtain error estimates for the elasto-plastic torsion, and Mosolov's problem.

(1) Manuscrit reçu le 6 février 1976.

( ${ }^{2}$ ) Dept. of Mathematics, Rutgers University, New Brunswick, U.S.A.

(3) Centre de Mathématiques Appliquées, École Polytechnique, Palaiseau,

$\left({ }^{*}\right)$ This work was supported under NSF grant, MPS 74-05795. 


\section{PHYSICAL PROBLeM}

Let us consider (as in [7]) a continuous medium $\Omega \subset \mathbf{R}^{N}$, submitted to body forces inside $\Omega$, and to pressure loads on a part $\Gamma_{F}$ of its boundary.

On the other part $\Gamma_{U}$, it is assumed to be fixed.

The stress field $\sigma \in K$, and the displacement field $u \in V$, are shown ([7]) to be solutions, if they exist, of the following relations :

$$
\begin{array}{ll}
(g(\sigma), \tau-\sigma)-(\varepsilon(u), \tau-\sigma) \geqslant 0 & \forall \tau \in K ; \\
(\sigma, \varepsilon(v))=L(v) & \forall v \in V ;
\end{array}
$$

with the following notation :

$$
V=\left\{v \in\left(H^{1}(\Omega)\right)^{N} \mid v=0 \text { on } \Gamma_{v}\right\}
$$

is the set of the admissible displacements.

$$
K=\{\tau \in Y \mid \tau(x) \in P \text { a.e. }\}
$$

is the convex set of plastically admissible stress fields, where

$$
Y=\left\{\tau \mid \tau_{i j} \in L^{2}(\Omega) ; \tau_{i j}=\tau_{j i} ; i, j=1, \ldots, N\right\}
$$

and $P$ is a fixed closed convex subset of $\mathbf{R}^{N^{2}}$.

We denote by $|$.$| the euclidean norm of \mathbf{R}^{N^{2}}$, and observe that $Y$ is a Hilbert space with the scalar product

$$
(\tau, \sigma)=\int_{\Omega} \sum_{i, j=1}^{N} \sigma_{i j} \tau_{i j} d x
$$

and associated norm

$$
\|\tau\|=\left(\int_{\Omega}|\tau|^{2} d x\right)^{1 / 2}
$$

$\varepsilon: V \rightarrow Y$ is the strain operator given by

$$
\varepsilon_{i j}(v) \equiv \frac{1}{2}\left(\frac{\partial v_{i}}{\partial x_{j}}+\frac{\partial v_{j}}{\partial x_{i}}\right)
$$

$L(v)$ is the work of the external loads in a "virtual " displacement $v \in V\left(L \in V^{\prime}\right)$.

$g: \mathbf{R}^{N^{2}} \rightarrow \mathbf{R}^{N^{2}}$ is an isomorphism representing the elasticity coefficients (the analogue of (1) in the elastic case would be $\varepsilon(u)=g(\sigma)$.

We make the following monotonicity hypothesis on $g$, i.e. there exists $\alpha>0$ such that

$$
J(\tau) \equiv \frac{1}{2}(g(\tau), \tau) \geqslant \alpha\|\tau\|^{2} \quad \forall \tau \in Y .
$$

We note this implies a coercivity condition on the "complementary energy" $J(\tau)$. 
Finally, we introduce the set of statically admissible stress fields

$$
M=\{\tau \in Y:(\tau, \varepsilon(v))=L(v), \forall v \in V\} .
$$

We choose $\tau \in K \cap M$ in (1). (We suppose the set $K \cap M$ is non empty.)

We then eliminate $u$, and we see that $\sigma$ is the solution of the problem $(P)$ : Find $\sigma \in K \cap M$ such that

$$
J(\sigma)=\inf _{\tau \in K \cap M} J(\tau) .
$$

Using hypothesis (3), we have the existence and uniqueness of $\sigma$. We are not able to prove, in the general case, that there exists a $u \in V$ such that $(\sigma, u)$ is a solution of (1), (2). However, in a slightly more restrictive case, we are able to prove the existence of a weak solution $u \in\left[L^{q^{\prime}}(\Omega)\right]^{N}$ (see section IV).

For the derivation of error estimates, we will assume that $u$ satisfies the regularity condition

$$
u \in V \cap\left[H^{2}(\Omega)\right]^{N}
$$

From the exact solutions, given by Mandel [14], we see that this hypothesis is not an unreasonable one, provided we are not near plastic collapse.

\section{APPROXIMATION}

Let us assume for simplicity that $\Omega$ is a bounded polytope. Corresponding to each value of a parameter $h, 0<h<1$, let $\mathscr{T}_{h}$ be a regular triangularization of $\Omega$ by $N$-simplices $T$ of sides less than or equal to $h$. Define $V_{h} \subset V$ as the subspace of functions in $V$ which are continuous on $\Omega$ and linear on each $T$ of $\mathscr{T}_{h}$, and $Y_{h} \subset Y$ as the subspace of tensors in $Y$ which are constant on each $T \in \mathscr{T}_{h}$. For properties of such finite element spaces, we refer the reader to [5], [6]. We note that

$$
\varepsilon: V_{h} \rightarrow Y_{h} \text {. }
$$

Using the above notation, we define our approximate problem $\left(P_{h}\right):$ Find $\sigma_{h} \in K \cap M_{h}$ such that

where

$$
J\left(\sigma_{h}\right)=\inf _{\tau_{h} \in K \cap M_{h}} J\left(\tau_{h}\right)
$$

$$
M_{h}=\left\{\tau_{h} \in Y_{h}:\left(\tau_{h}, \varepsilon\left(v_{h}\right)\right)=L\left(v_{h}\right), \forall v_{h} \in V_{h}\right\} .
$$

Applying the results of [16], we know that there exists a unique solution $\sigma_{h}$ to problem $\left(P_{h}\right)$ and that it converges to $\sigma$ as $h \rightarrow 0$. Our purpose, in this paper, is to derive an error estimate for $\left\|\sigma-\sigma_{h}\right\|$.

THEOREM 1: If $u \in\left[H^{2}(\Omega)\right]^{N}$, we have the error estimate

$$
\left\|\sigma-\sigma_{h}\right\| \leqslant C h\|u\|_{\left[H^{2}(\Omega)\right]^{N}}
$$

where $C$ is a constant independent of $h, u$, and $\sigma$. 
Proof : From (1), we get with $\tau=\sigma_{h}$

$$
\left(g(\sigma), \sigma_{h}-\sigma\right)-\left(\varepsilon(u), \sigma_{h}-\sigma\right) \geqslant 0
$$

and from the definition of $\sigma_{h}$, we have

$$
\left(g\left(\sigma_{h}\right), \tau_{h}-\sigma_{h}\right) \geqslant 0 \quad \forall \tau_{h} \in K \cap M_{h} .
$$

Writing $\tau_{h}-\sigma_{h}$ as $\tau_{h}-\sigma+\sigma-\sigma_{h}$, and adding (7) to (6), we get $\left(g\left(\sigma-\sigma_{h}\right), \sigma_{h}-\sigma\right)+\left(g\left(\sigma_{h}\right), \tau_{h}-\sigma\right)-\left(\varepsilon(u), \sigma_{h}-\sigma\right) \geqslant 0 \quad \forall \tau_{h} \in K \cap M_{h}$. Hence, applying (3)

$$
\alpha\left\|\sigma-\sigma_{h}\right\|^{2} \leqslant\left(g\left(\sigma_{h}\right), \tau_{h}-\sigma\right)-\left(\varepsilon(u), \sigma_{h}-\sigma\right) .
$$

Since $\sigma_{h} \in M_{h}$, and $\sigma \in M$, we have

so that

$$
\left(\sigma-\sigma_{h}, \varepsilon\left(v_{h}\right)\right)=0 \quad \forall v_{h} \in V_{h},
$$

$$
\left(\varepsilon(u), \sigma_{h}-\sigma\right)=\left(\varepsilon\left(u-v_{h}\right), \sigma_{h}-\sigma\right) \quad \forall v_{h} \in V_{h} .
$$

Since

$$
\begin{aligned}
\left(\varepsilon\left(u-v_{h}\right), \sigma_{h}-\sigma\right) & \leqslant\left\|\varepsilon\left(u-v_{h}\right)\right\|\left\|\sigma_{h}-\sigma\right\| \\
& \leqslant \frac{1}{2 \alpha}\left\|\varepsilon\left(u-v_{h}\right)\right\|^{2}+\frac{\alpha}{2}\left\|\sigma_{h}-\sigma\right\|^{2},
\end{aligned}
$$

we obtain, after collecting terms, that

$$
\begin{aligned}
\frac{\alpha}{2}\left\|\sigma-\sigma_{h}\right\|^{2} \leqslant\left(g\left(\sigma_{h}\right), \tau_{h}-\sigma\right)+\frac{1}{2 \alpha}\left\|\varepsilon\left(u-v_{h}\right)\right\|^{2}, & \\
& \forall v_{h} \in V_{h}, \quad \tau_{h} \in K \cap M_{h} .
\end{aligned}
$$

We now choose $\tau_{h}=\Pi_{h} \sigma$ where $\Pi_{h}$ denotes the projection of $Y \rightarrow Y_{h}$ associated with the norm $\|$.$\| . Then$

$$
\left(\sigma-\tau_{h}, \gamma_{h}\right)=0, \quad \forall \gamma_{h} \in Y_{h} .
$$

Applying (5) and using the fact that $\sigma \in M$, we see that

$$
\left(\tau_{h}, \varepsilon\left(v_{h}\right)\right)=\left(\sigma, \varepsilon\left(v_{h}\right)\right)=L\left(v_{h}\right) \quad \forall v_{h} \in V_{h},
$$

and hence $\tau_{h} \in M_{h}$. Since $Y_{h}$ is a space of piecewise constants,

$$
\left.\tau_{h}\right|_{T}=\frac{1}{\operatorname{meas}(T)} \int_{T} \sigma d x
$$

Then, since $\sigma \in P$ a.e., and $P$ is closed and convex, we get $\tau_{h} \in P$ for all $T \in \mathscr{T}_{h}$. Thus $\tau_{h} \in K \cap M_{h}$, and from (10),

$$
\left(g\left(\sigma_{h}\right), \tau_{h}-\sigma\right)=0 .
$$


Thus (9) becomes

$$
\left\|\sigma-\sigma_{h}\right\| \leqslant \frac{1}{\alpha}\left\|\varepsilon\left(u-v_{h}\right)\right\| \quad \forall v_{h} \in V_{h} .
$$

Using the continuity of $\varepsilon$ and the well known approximation properties of the space $V_{h}[5]$, we obtain

$$
\left\|\sigma-\sigma_{h}\right\| \leqslant C h\|u\|_{\left[H^{2}(\Omega)\right]^{N}}
$$

\section{REMARKS ON THE EXISTENCE OF A DISPLACEMENT}

As in [7], we make the following additional hypotheses. Let $\|\cdot\|$ be the $L^{\infty}$ norm defined by

We assume

$$
\|e\|_{\infty} \equiv \underset{x \in \Omega}{\operatorname{ess} \sup }|\mathrm{e}(x)|
$$

$\exists \delta>0$ and $\chi \in M$ such that $\chi+e \in K, \forall e \in Y$ with $\|e\|_{\infty} \leqslant \delta .(12)$

Furthermore, we shall restrict ourselves to the case where

$$
\Gamma_{F}=\varnothing \text { and where } L(v)=\int_{\Omega} f v d x, f \in\left[L^{q}(\Omega)\right]^{N} \text { with } q=N .
$$

Choosing $\chi_{h}=\Pi_{h} \chi$, we see that $\chi_{h} \in M_{h}$, and using the convexity of $P$, that $\chi_{h}$ belongs to the relative interior of $K$ in $Y_{h}$. We may then apply the Kuhn-Tucker theorem [18] to show the existence of $u_{h} \in V_{h}$ such that

$$
\left(g\left(\sigma_{h}\right), \tau_{h}-\sigma_{h}\right)-\left(\varepsilon\left(u_{h}\right), \tau_{h}-\sigma_{h}\right) \geqslant 0 \quad \forall \tau_{h} \in K .
$$

We now define $(D \tau)_{i}=-\sum_{j=1}^{N} \frac{\partial \tau_{i j}}{\partial x_{j}}$ and notice that $D: Y \rightarrow V^{\prime}$ is the adjoint
of $\varepsilon$.

Using the regularity we assumed on $L$, we see that the solution $\sigma$ of $(P)$ satisfies

$$
-D \sigma+l=0
$$

in the distribution sense on $\Omega$. Then

$$
\sigma \in K_{1}=\left\{\tau \in Y: D \tau \in\left[L^{q}(\Omega)\right]^{N}\right\} .
$$

We shall now prove the existence of a displacement $u$ which satisfies the following relation

$$
(g(\sigma), \tau-\sigma)-(u, D(\tau-\sigma)) \geqslant 0 \quad \forall \tau \in K_{1},
$$

which can be considered as a weak formulation of (1).

THEOREM 2 : Under hypothesis $(12)$, the sequence $\varepsilon\left(u_{h}\right)$ is bounded in $\left[L^{1}(\Omega)\right]^{N^{2}}$. Hence a subsequence of $u_{h}$ is converging weakly to $u \in\left[L^{q^{\prime}}(\Omega)\right]^{N}$ when $q^{\prime}=\frac{N}{N-1}$ and $(\sigma, u)$ is a solution of (14). 
Proof : Let $e \in Y$ satisfy $\|e\|_{\infty} \leqslant \delta$ and let $\chi$ be as defined in (12). Since $\tau_{h}=\Pi_{h} e+\chi_{h} \in K$, we may use this choise of $\tau_{h}$ in (13) to obtain $\left(g\left(\sigma_{h}\right), \Pi_{h} e\right)+\left(g\left(\sigma_{h}\right), \chi_{h}-\sigma_{h}\right)-\left(\varepsilon\left(u_{h}\right), \Pi_{h} e\right)-\left(\varepsilon\left(u_{h}\right), \chi_{h}-\sigma_{h}\right) \geqslant 0$.

Using the definition (10) of $\Pi_{h}$, we can replace $\Pi_{h} e$ by $e$ everywhere in (15). Since $\chi_{h}$ and $\sigma_{h} \in M_{h}$, the last term of (15) is zero. Applying the continuity of $g$, we get

$$
\left(e, \varepsilon\left(u_{h}\right)\right) \leqslant\left(g\left(\sigma_{h}\right), \chi_{h}-\sigma_{h}\right)+C \delta\left\|\sigma_{h}\right\|
$$

Since $\Omega$ is bounded, $\sigma_{h}$ being bounded in $Y$ implies $\sigma_{h}$ is also bounded in $\left[L^{1}(\Omega)\right]^{N^{2}}$. As $(16)$ is true for all $e \in Y$ with $\|e\|_{\infty} \leqslant \delta$, we get

$$
\left\|\varepsilon\left(u_{h}\right)\right\|_{\left[L^{1}(\Omega)\right]^{N^{2}}} \leqslant C \text {. }
$$

We then apply a result of Strauss [19] to obtain

$$
\left\|u_{h}\right\|_{\left[L^{q^{\prime}}(\Omega)\right]^{N^{2}}} \leqslant C\left\|\varepsilon\left(u_{h}\right)\right\|_{\left[L^{1}(\Omega)\right]^{N^{2}}} \leqslant C .
$$

From this, we deduce that a subsequence of $u_{h}$ (which we still denote by $u_{h}$ ) is converging weakly to $u$ in $\left[L^{q^{\prime}}(\Omega)\right]^{N}$.

For any $\tau \in K_{1}$, we choose $\tau_{h}=\Pi_{h} \tau$ in (13) and obtain

$$
\left(g\left(\sigma_{h}\right), \sigma_{h}\right) \leqslant\left(g\left(\sigma_{h}\right), \tau_{h}\right)-\left(\varepsilon\left(u_{h}\right), \tau_{h}-\sigma_{h}\right) .
$$

Now

$$
\left(\varepsilon\left(u_{h}\right), \tau_{h}\right)=\left(\varepsilon\left(u_{h}\right), \tau\right)=\left(u_{h}, D \tau\right) \rightarrow(u, D \tau),
$$

and since $\sigma_{h} \in M_{h}$,

$$
\left(\varepsilon\left(u_{h}\right), \sigma_{h}\right)=\left(f, u_{h}\right) \rightarrow(f, u)=(D \sigma, u) .
$$

Also

$$
\left(g\left(\sigma_{\tilde{n}}\right), \tau_{\dot{n}}\right)=\left(g\left(\sigma_{\dot{n}}\right), \tau\right) \rightarrow(g(\sigma), \tau),
$$

because $\sigma_{h}$ converges to $\sigma$, and $g$ is continuous. In the same way $\left(g\left(\sigma_{h}\right), \sigma_{h}\right)$ converges to $(g(\sigma), \sigma)$. Hence letting $h \rightarrow 0$ in (17), we obtain (14), which is the desired result.

\section{OTHER APPLICATIONS}

\subsection{Elastic-plastic torsion}

This problem is usually formulated as the following minimization problem, where $N=2,[7]$ :

Find $u \in K$ minimizing

$$
\begin{gathered}
\frac{1}{2}\|\nabla v\|^{2}-(f, v) \quad \text { over } K, \text { where } \\
K=\left\{v \in H_{0}^{1}(\Omega):|\nabla v| \leqslant 1 \text { a.e. in } \Omega\right\}, \text { and } \\
\|\cdot\|=\|\cdot\|_{\left[L^{2}(\Omega)\right]^{\lambda^{*}}} .
\end{gathered}
$$


Lemma 1 : Problem (18) is equivalent to the problem :

Find $p \in K_{1} \cap M$ minimizing $\frac{1}{2}\|p\|^{2}-(\varphi, p)$ over $K_{1} \cap M$, where $\varphi$ is any solution of $\operatorname{rot} \varphi \equiv \frac{\partial \varphi_{2}}{\partial x_{1}}-\frac{\partial \varphi_{1}}{\partial x_{2}}=-f$

$$
\begin{aligned}
& K_{1}=\left\{p \in\left[L^{2}(\Omega)\right]^{2}:|p| \leqslant 1 \text { a.e. in } \Omega\right\}, \text { and } \\
& M=\left\{p \in\left[L^{2}(\Omega)\right]^{2}:(p, \nabla \Psi)=0, \forall \Psi \in H^{1}(\Omega)\right\} .
\end{aligned}
$$

Proof: The result follows easily by using the fact that $p \in M$ is equivalent to $p=\operatorname{rot} v$ for some $v \in H_{0}^{1}(\Omega)$ (see [13]),

$$
\begin{aligned}
& (\varphi, \operatorname{rot} v)=(f, v), \quad \forall v \in H_{0}^{1}(\Omega) \\
& |\nabla v|=|\operatorname{rot} v| \quad \text { for } \quad v \in H_{0}^{1}(\Omega)
\end{aligned}
$$

(Recall that when $v$ is a scalar, rot $v$ is the vector deduced from the gradient by a rc ation of $+\frac{\pi}{2}$ )

REMARK : We note that problem (19) is in fact the original problem (see [7]). We further note that problem (19) can be derived from the more general problem :

Find $(\cdot, \chi) \in\left(K_{1} \cap M\right) \times H^{1}(\Omega)$ satisfying

$$
(p, q-p)-(\varphi+\nabla \chi, q-p) \geqslant 0 \quad \forall q \in K_{1} \text {. }
$$

Using a result of Brezis [2], it was proved in [15] that there exists a solution to problem (20), when $f$ is constant.

We will assume, as in section II, that $\chi$, which may be interpreted as a displacement, belongs to $H^{2}(\Gamma)$ We know from [3] that $p \in\left[H^{1}(\Omega)\right]^{2}$ for $f \in L^{2}(\Omega)$.

Following the ideas of se: on III, we approximate problem (19) by the problem

Find $p_{h} \in K_{1} \cap M_{h}$ minimizing

$$
\begin{gathered}
\frac{1}{2}\left\|p_{h}\right\|^{2}-\left(\varphi, p_{h}\right) \text { over } K_{1} \cap M_{h}, \text { where } \\
M_{h}=\left\{p_{h} \in Y_{h}:\left(p_{h}, \nabla \Psi_{h}\right)=0 \forall \Psi_{h} \in V_{h}\right\},
\end{gathered}
$$

$Y_{h}$ is the subspace of $\left[L^{2}(\Omega)\right]^{2}$ of piecewise constants, and

$V_{h}$ is the subspace of $H^{1}(\Omega)$ of continuous piecewise linear functions.

vol. $11, n^{\circ} 2,1977$. 
THEOREM 3 : If $\varphi \in\left[H^{1}(\Omega)\right]^{2}$ and $\chi \in H^{2}(\Omega)$, then we have the error estimate $\left\|p-p_{h}\right\| \leqslant C h\left[\|\varphi\|_{1}+\|\chi\|_{2}\right]$

where 'is a constant independent of $\varphi, \chi$ and $h .\left(\|\varphi\|_{1}\right.$ is the norm of $\varphi$ in $\left[H^{1}(\Omega)\right\rfloor^{2}$ and $\|\chi\|_{2}$ is the norm of $\chi$ in $H^{2}(\Omega)$ ).

Proof : Proceeding i $n$ identical fashion to the proof of theorem 1, we easily obtain the estir.late

$$
\frac{1}{2}\left\|p-p_{h}\right\|^{2} \leqslant \frac{1}{2}\left\|\nabla\left(\chi-\chi_{h}\right)\right\|^{2}+\left(\varphi, p-q_{h}\right) \quad \forall \chi_{h} \in V_{h},
$$

where $q_{h}$ has been chosen as the $\left[L^{2}(\Omega)\right]^{2}$ projection of $p$ onto $Y_{h}$. Since

$$
\left(\varphi_{h}, p-q_{h}\right)=0 \quad \forall \varphi_{h} \in Y_{h},
$$

we $g$ t

$$
\begin{aligned}
\left(\varphi, p-q_{h}\right)=\left(\varphi-\varphi_{h}, p-q_{h}\right) & \leqslant\left\|\varphi-\varphi_{h}\right\|\left\|p-q_{h}\right\| \\
& \leqslant C h_{2}\|\varphi\|_{1}\|p\|_{1}
\end{aligned}
$$

(using the standard approximation properties of $Y_{h}$ and the assumed regularity of $p$ and $\varphi$ ). Estimating

$$
\left\|\nabla\left(\chi_{h}-\chi\right)\right\|^{2} \leqslant C h^{2}\|\chi\|_{2}^{2}
$$

as before, we obtain the desired result.

We remark that the approximation given by (21) is not equivalent to the usual direct approximation of problem (18) by piecewise linear finite elements [10], since this would lead to an internal approximation of $M$, which is not the case here $\left(M_{h} \not \subset M\right)$. For the direct approximation, non-optimal error estimates have previousiy been derived in $[\delta]$.

\subsection{Mosolov's problem [7]}

This problem is usually formulated as the following :

Find $u \in H_{0}^{1}(\Omega)$ minimizing

$$
\begin{gathered}
\frac{1}{2}\|\nabla v\|+j(\nabla v)-(f, v) \text { over } H_{0}^{1}(\Omega), \text { where } \\
j(p) \equiv g \int_{\Omega}|p| d x .
\end{gathered}
$$

Since $\Omega \subset \mathbf{R}^{2}$, we form an equivalent problem in a similar fashion to lemma 1. We get problem

Find $p \in M$ minimizing

$\frac{1}{2}\|q\|^{2}+j(q)-(\varphi, q)$ over $M$, where $\varphi$ and $M$ are chosen as in section $5 \cdot 1$. 
Using duality theory, we have that problem (23) is the dual of the problem

$$
\sup _{\psi \in H^{1}(\Omega)}-\frac{1}{2}\left\|\{|\varphi+\nabla \Psi|-g\}^{+}\right\|^{2} \quad \text { (see [17]). }
$$

Since the problem is coercive in $H^{1}(\Omega) / \mathbf{R}$, we know that it has a solution $\chi \in H^{1}(\Omega)$. Hence $(p, \chi)$ satisfies the following extremality relation

$$
(p, q-p)+j(q)-j(p)-(\varphi+\nabla \chi, q-p) \geqslant 0 . \quad \forall q \in\left[L^{2}(\Omega)\right]^{2} .
$$

We will again assume that $\chi \in H^{2}(\Omega)$, which is a valid assumption at least for the exact solution computed by Glowinski [9]. Using our general technique once more we approximate (23) by the following problem.

Find $p_{h} \in M_{h}$ minimizing

$$
\frac{1}{2}\left\|q_{h}\right\|^{2}+j\left(q_{h}\right)-\left(\varphi, q_{h}\right) \quad \text { over } \quad q_{h} \in M_{h},
$$

where $M_{h}$ is defined as in section 5.1.

THEOREM 4 : If $\varphi \in\left[H^{1}(\Omega)\right]^{2}$ and $\chi \in H^{2}(\Omega)$, then we have the error estimate

$$
\left\|p-p_{h}\right\| \leqslant C h\left[\|\varphi\|_{1}+\|\chi\|_{2}\right]
$$

Proof : Proceeding in an identical fashion to the proof of theorem 3, we easily obtain the estimate

$$
\frac{1}{2}\left\|p-p_{h}\right\|^{2} \leqslant C h^{2}\left[\|\varphi\|_{1}+\|\chi\|_{2}\right]^{2}+j\left(q_{h}\right)-j(p),
$$

where $q_{h}$ is again the $\left[L^{2}(\Omega)\right]^{2}$ projection of $p$ onto $Y_{h}$. Hence

$$
\left.\forall T \in \mathcal{C}_{h} \quad q_{h}\right|_{T}=\frac{1}{\operatorname{meas}(T)} \int_{T} p d x,
$$

and the convexity of $j$ implies that $j\left(q_{h}\right) \leqslant j(p)$. Thus, we get the desired result.

We remark that this approximation is again different from the direct approximation of (22) for which quasi-optimal error estimates have already been derived [9].

As far as we know, the approximate problem (25) has not been solved numerically. What we should suggest for such a $\mathrm{n}$.merical computation is to try to solve directly the approximation of the dual problem (24), when $H^{1}(\Omega)$ is approximated by $V_{h}$, because this problem would be the dual of (25). Furthermore, it is a problem of unconstrained minimization of a differentiable (but not strictly convex) function.

\section{ACKNOWLEDGEMENTS}

We wish to thank Professors Nedelec and Raviart for several helpful discussions. vol. $11, \mathrm{n}^{\circ} 2,1977$. 


\section{REFERENCES}

1. H. Brezis, Monotonicity in Hilbert spaces and some applications to nonlinear partial differential equations. Contributions to nonlinear functional analyzis, E. Zarantonello (ed.), Academic Press, New York, 1971, pp. 101-156.

2. H. BREZIS, Multiplicateur de Lagrange en torsion élasto-plastique, Arch. Rat. Mech. Anal., 2972, pp. 32-40.

3. H. Brezis, G. Stampacchia, Sur la régularité de solutions d'inéquations elliptiques, Bull. Soc. Math. France, Vol. 96, 1968, 153-180.

4. F. Brezzi, W. HAGER, P. A. RAVIART, Error estimates for variational inequalities, to appear.

5. P. G. Ciarlet, Numerical analysis of the finite element method, Cours d'été d'Analyse Numérique, 1975, Université de Montréal.

6. P. G. CIARLET, The finite element method, North Holland (to appear).

7. G. Duvaut, J. L. Lions, Les inéquations en Mécanique et en Physique, Dunod, Paris, 1972.

8. R. S. FALK, Approximate solutions of some variational inequalities with order of convergence estimates, Ph. D. Thesis, Cornell University, Ithaca, N. Y., 1971.

9. R. GLowINSKI, Sur l'approximation d'une inéquation variationnelle elliptique de type Bingham, R.A.I.R.O., Analyse Numérique Vol. 10, 12 (1976) 13-30.

10. R. Glowinski, J. L. Lions, R. Tremolieres, Approximation des inéquations variationnelles, Dunod, Paris, 1976.

11. C. Johnson, Existence theorems for plasticity problems, J. Math. Pures et Appliquées, 55 (1976) 431-444.

12. C. JoHnson, $A$ hybrid finite element method for plasticity problems with hardening, to appear.

13. O. A. LADYZENSKAYA, The mathematical theory of viscous incompressible flow, Gordon \& Breach, New York, 1969.

14. J. Mandel, Cours de Mécanique des Milieux Continus, T. 2, Mécanique des Solides, Gauthier-Villars, Paris, 1966.

15. B. MERCIER, Recherche numérique d'un champ de déplacements en élasto-plasticité, Rapport Laboria $\mathrm{n}^{\circ}$ 71, Iria, 78150 Le Chesnay, France, 1974.

16. B. MERCIER, Approximation par éléments finis, et résolution par un algorithme de pénalisation-dualité d'un problème d'élasto-plasticité, C. R. Acad. Sc. Paris, T. 280, Série A, 1975, pp. 287-290.

17. B. MERCIER, Une méthode de résolution du problème des charges limites utilisant les fluides de Bingham, C. R. Acad. Sc. Paris, T. 281, Série A, 1975, pp. 525-527.

18. R. T. Rockafellar, Convex analysis, Princeton, corollary 28-2-2, 1970, p. 279.

29. M. J. Strauss, Variations of Korn's and Sobolev's inequalities, Proc. of Symposia in Pure Math., Vol. XXIII, A.M.S., Providence, R. I., 1973, pp. 207-214. 